\title{
Sensing and responding to hypersaline conditions and the HOG signal transduction pathway in fungi isolated from hypersaline environments: Hortaea werneckii and Wallemia ichthyophaga
}

\section{Ana Plemenitaš}

Institute of Biochemistry and Molecular biology, Faculty of Medicine, University of Ljubljana, Vrazov trg 2, Ljubljana, Slovenia

\begin{abstract}
Sensing and responding to changes in $\mathrm{NaCl}$ concentration in hypersaline environments is vital for cell survival. We have identified and characterized key components of the high-osmolarity glycerol (HOG) signal transduction pathway, which is crucial in sensing hypersaline conditions in the extremely halotolerant black yeast Hortaea werneckii and in the obligate halophilic fungus Wallemia ichthyophaga. Both organisms were isolated from solar salterns, their predominating ecological niche. The identified components included homologous proteins of both branches involved in sensing high osmolarity (SHO1 and SLN1) and the homologues of mitogen-activated protein kinase module (MAPKKK Ste11, MAPKK Pbs2, and MAPK Hog1). Functional complementation of the identified gene products in S. cerevisiae mutant strains revealed some of their functions. Structural protein analysis demonstrated important structural differences in the HOG pathway components between halotolerant/halophilic fungi isolated from solar salterns, saltsensitive $S$. cerevisiae, the extremely salt-tolerant $H$. werneckii, and halophilic $W$. ichthyophaga. Known and novel gene targets of MAP kinase Hog1 were uncovered particularly in halotolerant H. werneckii. Molecular studies of many salt-responsive proteins confirm unique and novel mechanisms of adaptation to changes in salt concentration.
\end{abstract}

Keywords: Halotolerant/halophilic fungi; Hortaea werneckii; Wallemia ichthyophaga; HOG signal transduction Pathway

\section{Introduction}


Solar salterns and other hypersaline environments are extreme habitats that prevent the growth of most organisms except those that are well adapted to extremely high salt concentrations. Our laboratory has long been involved in studies of the molecular mechanisms of adaptations to extremely high $\mathrm{NaCl}$ concentrations We predominantly use two fungal model organisms isolated from solar salterns: the extremely halotolerant Hortaea werneckii and the obligate halophilic fungus Wallemia ichthyophaga (Gunde-Cimerman et al. 2009, 2014 and 2018; Petrovič et al.2002; Plemenitaš et al. 2014; Zajc et al. 2014). While H. werneckii is unique in its adaptability to fluctuations in salt concentration and grows without $\mathrm{NaCl}$ as well as in the presence of up to $5 \mathrm{M}$ $\mathrm{NaCl}, W$. ichthyophaga is a true halophile that requires the presence of at least $10 \%$ of $\mathrm{NaCl}$ and also grows in the presence of up to $32 \% \mathrm{NaCl}$. Whole genome sequencing of both fungi, ion measurements, and studies of the adaptations of numerous proteins and membrane properties to high $\mathrm{NaCl}$ concentrations have revealed many new molecular mechanisms of adaptation. Some of these mechanisms are common to both fungi, but many are unique to halotolerant $H$. werneckii and halophilic W. ichthyophaga. Sensing changes in salt concentrations, which leads to appropriate responses regarding the expression of osmoresponsive genes, is vital for survival in environments with increased $\mathrm{NaCl}$ concentrations.

We were therefore particularly interested in the high-osmolarity glycerol (HOG) signal transduction pathway, which is involved in sensing hyperosmolar conditions. Identifying components of the HOG pathway in $H$. werneckii and W. ichthyophaga and investigating their roles in the adaptation to hypersaline conditions demonstrated that both fungi are well adapted to such conditions. Furthermore, both fungi sense and respond to increased environmental salinity differently. In this review article, we summarize the key molecular adaptations of $H$. werneckii and W. ichthyophaga, focusing on the identification and structural properties of HOG pathway components.

\section{Molecular adaptations of halotolerant $\mathrm{H}$. werneckii}

The extremely halotolerant black yeast H. werneckii (Capnodiales, Dothideomycetidae, Dothideomycetes, Pezizomycotina) is described as an opportunistic pathogen and the primary etiological agent of tinea nigra, which can occur on the salty hands and feet of humans. It has been isolated from different hypersaline environments, with brine in solar salterns representing its 
primary ecological niche (Butinar et al. 2005; Gunde-Cimerman and Zalar 2014). H. werneckii is an example of a very adaptable fungal species, as it can grow without salt and also in environments with extremely high $\mathrm{NaCl}$ concentrations (up to $30 \%$ ).

Molecular studies have identified general and also unique molecular mechanisms of adaptation that contribute to the extreme halotolerance of $H$. werneckii (Petrovič et al 2002, Gunde-Cimerman at al.2009 and 2018; Plemenitaš et al. 2014). The most striking result came from the whole genome sequencing of $H$. werneckii, which was also the first published whole genome sequence of a fungal species isolated from an environment with an extremely high $\mathrm{NaCl}$ concentration. With almost 50 $\mathrm{Mb}$, the $H$. werneckii genome is larger than the known genomes of other Capnodiales representatives, and, unlike in other species with large genomes, the large genome of $H$. werneckii is not due to large amounts of repeated DNA content. Analysis demonstrated recent duplication of the $H$. werneckii genome, with multiple copies of halotolerance genes, in particular genes that code for metal cation transporters (Lenassi at al. 2013, Sinha et al. 2017). The H. werneckii genome contains almost 16,000 genes of which $90 \%$ are duplicates with an average protein sequence divergence of 5\%. While post-duplication events normally include large-scale gene deletions and reductions, the whole genome duplication appears stable in $H$. werneckii, as the genome was not reduced even after long-term growth in the presence of salt stress (Sinha et al. 2017, Gostinčar et al. 2021).

These whole genome data fit well with the results of molecular studies on individual genes. Most of the studied genes at the protein level are duplicated and code for two functionally identical proteins, often with a salt-dependent expression pattern. Such proteins are involved in general responses, as exemplified by the salt-dependent expression of two forms of glycerol phosphate dehydrogenase, which are involved in the synthesis of glycerol (the main compatible solute in $H$. werneckii) (Lenassi et al. 2011), fatty acid desaturases and elongases, which are involved in saltdependent changes of membrane phospholipids (Gostinčar et al. 2009; Turk et al. 2004 and 2011), and especially metal cation transporters that are not only duplicated but exist as multiple copies in the $H$. werneckii genome (Lenassi et al. 2013). The abundance of cation transporters in the $H$. werneckii genome indicates the importance of regulating potassium and sodium transport across the plasma membrane. Enrichment was not only observed for the transporters that maintain high intracellular $\mathrm{K}^{+} / \mathrm{Na}^{+}$ratios and participate in $\mathrm{Na}^{+}$efflux and $\mathrm{K}^{+}$influx (e.g., ENA, NHA, and TRK) 
at high $\mathrm{NaCl}$ concentrations, but also for those involved in $\mathrm{K}^{+}$efflux $(\mathrm{TOK})$ and $\mathrm{Na}^{+}$influx $(\mathrm{PHO})$ at low $\mathrm{NaCl}$ concentrations (Kogej et al 2005, Gorjan and Plemenitaš 2006, Lenassi et al 2013, Plemenitaš et al, 2016). This fits well with the lifestyle of $H$. werneckii, which thrives in environments with fluctuations in $\mathrm{NaCl}$ concentration, as maintenance of appropriate $\mathrm{K}^{+} / \mathrm{Na}^{+}$ratios is not crucial only at high $\mathrm{NaCl}$ concentrations but also at low $\mathrm{NaCl}$ concentrations

Two proteins with a specific biochemical signature of halophily were also identified in $H$. werneckii: Hydroxymethylglutaryl CoA (HMG-CoA) reductase), the main regulatory enzyme in the mevalonate pathway (Vaupotič and Plemenitaš 2007, Vaupotič et al 2008), and 3'phosphoadenosine-5'-phosphatase encoded by the HAL2 gene, a known target of salt toxicity in yeast (Vaupotič et al. 2007a). NaCl-dependent regulation of HMG-CoA reductase in H. werneckii also appears to be linked to its halotolerant character. Both enzyme activity and protein levels in $H$. werneckii depend on environmental salinity, with high levels under both hypo- and hyper-saline conditions. Interestingly, we found that in $\mathrm{H}$. werneckii, the biological consequence of HMG-CoA regulation relates to posttranslational protein modification by prenylation and not to sterol synthesis, as shown, for example, in S. cerevisiae. Thus, HMG-CoA reductase can be considered an important biochemical signature of halophilism. In evolutionary terms, the maintenance of high HMG-CoA reductase levels in hypo- and hyper-saline environments may also reflect the physiological adaptation of halophilic fungi to metabolic demands under extreme conditions (Vaupotoč et al 2008).

Another example of a protein with specific responses to changes in salinity is 3'phosphoadenosine-5'-phosphatase Hal2. Two isoforms of the Hal2 protein appear to significantly contribute to the adaptation of $H$. werneckii to fluctuations in environmental salinity. With genetic and biochemical validation, the specific sequence motif of Hal2, which we called the META sequence, was identified to exert an effect on salt tolerance in the HwHal2B isoform as well as when introduced into the SAL1 gene in Arabidopsis thaliana (Vaupotič et al. 2007a, Buh Gašeršič et al 2013).

\section{The HOG signal transduction pathway in halotolerant $H$. werneckii}


The HOG signal transduction pathway is an example of a MAP kinase (mitogen-activated protein kinase) signal transduction pathway that senses and responds to hyperosmolar conditions and thus plays an important role in the adaptation to hypersaline conditions. It consists of the proteins of the sensory apparatus and the module of three kinases: MAPKKK (mitogen-activated protein kinase kinase kinase), MAPKK (mitogen-activated protein kinase kinase), and MAPK (mitogenactivated protein kinase); these are activated by sequential phosphorylation. This mechanism is best understood in the yeast $S$. cerevisiae. Hyperosmolar signals are sensed and transduced by two branches in S. cerevisiae: SHO1 and SLN1 that converge in the activation of MAPKK ScPbs2. In the SHO1 branch, the transmembrane ScSho1, membrane anchor ScOpy2, mucines ScMsb2 and ScHkr1, guanine exchange factor ScCdc24, and GTPase ScCdc42 are involved in the SHO1 sensory apparatus, which leads to the activation of ScSte20. Consequently, MAPKKK ScSte11 is activated by phosphorylation by the ScSte20 kinase. In the SLN1 branch, the proteins Sln1, Ypd1, and Ssk1 form the two-component phosphorelay system in which the sensor histidine kinase ScSln1 (located in the plasma membrane) is inactivated under hyperosmolar conditions. The ScYpd1 intermediate protein and the ScSsk1 response regulator then transduce the signal to the MAPKKKs ScSsk2/ScSsk22. The MAPKKKs ScSte11 and ScSsk2/ScSsk22 activate the MAPKK $\mathrm{ScPbs} 2$, which serves as a scaffold protein of the two upstream pathway branches and upon phosphorylation activates ScHog1 MAPK (Hohmann et al., 2007, Hohmann 2009, Saito and Posas, 2012, Tatebayashi et al., 2015). Under hyperosmotic conditions, ScHog1 phosphorylation is accompanied by an import of ScHog1 into the nucleus, which leads to the regulation of osmoresponsive genes. In addition, activated ScHog1 has targets in the cytosol and plasma membrane (Hohmann 2009) that also play a role in osmoadaptation. The phosphorylation state of the MAPK Hog 1 is transient and is controlled by various phosphoprotein phosphatases, like Ser/ Thr phosphatase Ptc1 (Warmka et al 2001 ) and phosphotyrosine phosphatases Ptp2 and Ptp3 (Jacoby et al. 1997).

Homologous components to the $S$. cerevisiae HOG pathway have also been identified in other yeasts and fungi and are involved in responses to hyperosmolar conditions, virulence regulation, gut colonization, and animal and plant infection with pathogenic fungi. According to published data, it seems that two-component phosphorelay systems play important roles in sensing and subsequent HOG pathway activation in different fungi. For example, in the filamentous fungi 
Aspergillus fumigatus and Aspergillus nidulans, the HOG pathway is involved in osmotic stress responses (Ma and Li, 2013; de Oliveira Bruder Nascimento et al., 2016), and the Sho1 protein is not involved in osmosensing in A. nidulans (Furukawa et al., 2005). Likewise, the HOG pathway in the pathogenic fungus Cryptococcus neoformans is activated exclusively via the two-component phosphorelay system (Bahn et al., 2006). Histidine kinases were also identified as the main regulators of the HOG signal transduction pathway in Magnaporthe oryzae (Jacob et al., 2015) and Candida albicans (Prieto et al., 2014).

Since the HOG pathway is highly conserved among eukaryotes, similar components to those in $S$. cerevisiae were expected in $H$. werneckii. Indeed, the genome of $H$. werneckii contains all the key components of the HOG signal transduction pathway. In line with the genome duplication in $H$. werneckii, we identified two copies of each gene that code for the components of the HOG pathway( (Lenassi et all 2013, Sinha et all 2017 (Fig. 1). As there are no genetic tools available to allow manipulation of the $H$. werneckii genome, the putative roles of HOG pathway components identified in $H$. werneckii were studied by functional complementation in $S$. cerevisiae mutant strains (Turk et all. 2002, Fettich et all.2011, Kejžar et all 2015).

\subsection{The sensory apparatus of the HOG pathway in H.werneckii}

Although important differences were found in the sensory apparatus when compared to $S$. cerevisiae, it appears that, in contrast to other studied fungi, both the SLN1 and SHO1 branches play a role in sensing hypersaline conditions in $H$. werneckii, as we were able to identify components of both branches in $H$. werneckii. Interestingly, besides the SLN1 gene, which codes for membrane-bound histidine kinase $\operatorname{Sln} 1$, the genome of $H$. werneckii contains two additional histidine kinases genes, $H w N I K 1$ and $H w H H K 7$, which code for cytosolic histidine kinases Nik1 and Hhk7 Lenassi at all 2013, Lenassi at all 2006). While the role of membrane-bound Sln1 in sensing hyperosmolar conditions is well documented in S. cerevisiae, the role of the cytosolic histidine kinases Nik1 and Hhk7 is less understood. Both were identified and proposed to be involved in sensing hyperosmolar conditions in pathogenic fungi (Furukawa at all 2005, Radhawa et all 2016)) 
We identified two isoforms of histidine kinase HwHhk7 in H. werneckii: HwHhk7A and B (Fig. 1 (Lenassi 2006)). Phylogenetic comparisons with other fungal histidine kinases (HK) and analyses of structural motifs confirmed that they belong to group 7 of fungal HKs. According to published data (Catlett et al 2003), histidine kinases Sln1 and HK 7 groups are positioned close together, and thus late separation from a common ancestor was proposed. However, these proteins differ in their intracellular localization: while histidine kinases from the Sln1 group are membranebound, histidine kinases from the HK7 group are soluble cytosolic proteins. It has been proposed that some of the histidine kinases other than yeast orthologs might transmit the signal through Ypd1; for example, in A. nidulans, the HOG pathway was reconstructed and revealed that the Sho1 branch is not involved in the osmoresponsive activation of the MAPK Hog1. It was speculated that this role could well be occupied by the HK 7 protein AnM7 (Han and Prade 2002, Furukawa et al., 2005).

To obtain insight into the role of the HwHhk7 isoforms in response to changes in salinity in halotolerant $H$. werneckii, we assessed the expression of both isoforms in response to changes in $\mathrm{NaCl}$ concentration. We found that the expression of the $H w H H 7 A$ gene did not change significantly with changes in $\mathrm{NaCl}$ concentration. By contrast, transcription of the $H w H H K 7 B$ gene was very salt-responsive. Moreover, we observed two types of responses: an early response with high induction of gene expression under hyposaline conditions and a late increased expression of the $H w H H 7 B$ gene under hypersaline stress (Lenassi et al 2006). Based on these results, we proposed that in $H$. werneckii, the high induction of $H w H H K 7 B$ gene expression as an early response to hyposaline stress could be the result of the specialized role of this HK in response to conditions of modest osmolarity, as has already been demonstrated for the $\operatorname{Sln} 1 \mathrm{HK}$ in S. cerevisiae (O'Rourke and Herskowitz, 2004). H. werneckii is extremely adaptable to changes in $\mathrm{NaCl}$ concentration. Salt-dependent expression of the $H w H H 7 B$ gene suggests that HwHhk7B plays an important role in sensing and adapting to the sudden changes that are very common in solar salterns, natural habitats of $H$. werneckii.

Analysis of the $H$. werneckii genome also confirmed the presence of genes that code for proteins of the sensory apparatus of the SHO1 branch, membrane-bound HwSho1 A and B, HwOpy2 A and B, and HwMsb2 A and B. Structural and functional studies were performed on HwSho1 (Fettich et al. 2011). Comparison of amino-acid sequences of HwSho1 A and B in extremely 
halotolerant $H$. werneckii with those of salt-sensitive $S$. cerevisiae, $C$. albicans, and $A$. fumigatus and moderately halotolerant Debaryomyces hansenii showed highly conserved transmembrane and SH3 domains and a poorly conserved linker domain containing the Ste11-binding site. Despite differences in the Ste11-binding domain between $H$. werneckii and S. cerevisiae Sho1 proteins, both HwSho1 isoforms fully complemented the function of the native S. cerevisiae Sho1 protein when expressed in the $S$. cerevisiae strain with the deleted SHOI gene. Furthermore, they both activated the HOG pathway under conditions of osmotic stress. Moreover, we demonstrated that in $S$. cerevisiae, both HwSho1 proteins have characteristic subcellular localizations similar to the Sho1 protein (Fettich et al 2011). These data suggest that HwSho1 is involved in sensing hypersaline stress conditions in $H$. werneckii.

It appears that the sensory apparatus in the $H$. werneckii HOG pathway is much more complex when compared to $S$. cerevisiae and other plant and human pathogenic fungi. Based on our studies, we propose that the signaling to HwPbs2 in H. werneckii is conveyed through both branches: (1) the SHO1 branch, which is similar to that in S. cerevisiae, and (2) the SLN1 branch, which is similar to that in pathogenic fungi such as A. nidulans and C. albicans in which histidine kinases were proposed to be responsible for transmitting the signal to the YpdA-SskA-SskB phosphorelay. We can speculate that the combination of both sensory systems in sensing changes in environmental salinity enables a more fine-tuned response to osmolarity in $H$. werneckii.

\subsection{The MAP kinase module of the HOG pathway in H.werneckii}

Besides the genes of the sensory apparatus in the $H$. werneckii genome, gene duplication was also confirmed for downstream HOG pathway key components, including HwSTE20, HwSTE50, and genes that code for the three kinases of the MAP kinase module (HwSTE11 A and B, HwPBS2 A and $\mathrm{B}$, and HwHOG1 A and B). Further structural and functional studies of HwPbs2 and HwHog1 uncovered unique features of these kinases. Interestingly, in the case of the MAPKK HwPbs2, two gene copies of $H W P B S 2 \mathrm{~A}$ and $\mathrm{B}$ are transcribed and translated into three different isoforms: while $H w P B S 2 A$ is translated into one protein product (HwPbs2A), HwPBS2B is translated into two protein products (HwPbs2B1 and $\mathrm{HwPbs} 2 \mathrm{~B} 2)$. Multiple isoforms were confirmed by Western blot analysis with specific antibodies raised against $\mathrm{HwPbs} 2$. It was found that they respond differently to changes in salt concentration. Their salt-responsive expression profiles suggest that $\mathrm{HwPbs} 2 \mathrm{a}$ 
and $\mathrm{HwPbs} 2 \mathrm{~b} 2$ play roles in quick adaptation to severe hypersaline shock and that HwPbs $2 \mathrm{~b} 1$ plays a role in adaptation to moderate salt stress (Lenassi, Doctoral thesis, unpublished data).

The $H$. werneckii HOG signal transduction pathway includes two functionally redundant MAPK homologues, HwHog1A and HwHog1B. To better understand the function of the HOG pathway in $H$. werneckii, we observed the osmotic stress responsive phosphorylation pattern of HwHog1 and the effect of the Hog1 kinase inhibitor on cell viability and survival under hypersaline conditions. Activation of the HOG pathway in $H$. werneckii in response to increased osmolarity revealed that HwHog1A and HwHog1B are fully activated by constitutive phosphorylation with the upstream kinase HwPbs2 only at concentrations above 17\% NaCl (Kejžar et al 2015a). Thus, while phosphorylation is transient for most fungal species, this does not appear to be the case in extremely halotolerant $H$. werneckii. Likewise, the effect of HwHog1 kinase activity inhibition on restricting $H$. werneckii colony growth was only observed at osmolyte concentrations of $\geq 17 \%$.

Studies on the $H$. werneckii transcriptome revealed many novel osmoresponsive genes, most of which were not shown before for S. cerevisiae or other fungi. Direct interaction with the MAP kinase HwHog 1 was demonstrated for more than one third of salt-responsive genes (Fig. 1), with majority of them upregulated in high-salinity-adapted H. werneckii cells (Vaupotič et al 2007). Genes associated with energy supply were highly represented among the upregulated genes in cells growing at $30 \% \mathrm{M} \mathrm{NaCl}$. An increase in energy production appears to be one of the fundamental adaptations that maintain ion homeostasis and osmotic equilibrium in a hyperosmotic environment. During long-term adaptive growth under extreme salinity, $H$. werneckii must maintain a high production of ATP that powers various transmembrane transporters (Petrovič et al. 2002, Vaupotič et al. 2007, Gostinčar et al. 2011). In contrast to constitutive phosphorylation of $\mathrm{HwHog} 1$ in response to $\mathrm{NaCl}$, the activation of osmoresponsive gene transcription was transient, which suggests that other factors besides the HwHog1 phosphorylation signal are involved in the effects of nuclear HwHog1 in H. werneckii (Kejžar et al 2015).

We also showed that $H$. werneckii can discriminate between different osmolytes. $\mathrm{NaCl}$ induces continuous phosphorylation of HwHog1, whereas this phosphorylation is transient in response to $\mathrm{KCl}$ or sorbitol. Discrimination between osmolyte types was also confirmed at the level of osmoresponsive gene transcription. In H. werneckii cells, genes such as HwSTL1 and HwGPD1, which are involved in glycerol transport and synthesis, showed early induction of transcription in 
response to $\mathrm{NaCl}$ stress, early or late induction in response to sorbitol stress, and no effects in response to $\mathrm{KCl}$ stress (Kejžar et al 2015). This osmolyte-specific response was also demonstrated in the expression of genes related to mitochondrial function. In hypersaline medium, an increased expression of genes involved in energy production and oxidative damage protection was observed, whereas adaptation to a non-ionic osmolyte resulted in a decrease in ATP synthesis and lipid peroxidation levels in mitochondria. This was also confirmed with a proteomic study of mitochondrial proteins, revealing a preferential accumulation of energy metabolism enzymes in hypersaline medium and an accumulation of protein chaperones in the non-ionic osmolyte (Vaupotič et al. 2007 and 2008).

In summary, the extremely halotolerant yeast-like fungus $H$. werneckii, isolated from hypersaline saltern water, can grow in both environments without salt as well as with extremely high salt concentrations (even up to saturated $\mathrm{NaCl}$ solutions). We assume that this extremely adaptable fungus has an efficient sensory system and molecular machinery that responds to changing conditions in the environment. As a response to severe changes in salinity in the environment, $H$. werneckii activates the HOG signaling pathway, resulting in the expression of many saltresponsive genes. The HOG signal transduction pathway is vital for the extreme osmotolerance of H. werneckii and regulates common and osmolyte-specific processes. Particularly, the HOG pathway seems to be specifically responsive to $\mathrm{Na}^{+}$, an ion typically present at high concentrations in the natural habitat of $H$. werneckii, solar eutrophic salterns.

\section{Molecular adaptations of the halophilic fungus W. ichthyophaga}

W. ichthyophaga (Wallemiales, Wallemiomycetes) is a xerophilic filamentous fungi. Although xerotolerance is rare in Basidiomycota, W. ichthyophaga is the most halophilic fungus known. It requires a minimum of $10 \%(\mathrm{w} / \mathrm{v}) \mathrm{NaCl}$, with a growth optimum at $15-20 \% \mathrm{NaCl}$, but is metabolically active even at $32 \% \mathrm{NaCl}$ (Zajc et al. 2014). Strains of W. ichthyophaga have been isolated from hypersaline waters of solar salterns, bitterns, and salted meat ( Jančič et al. 2015 and 2016). Morphological studies of W. ichthyophaga have shown that changes in the size of cell aggregates and the thickness of the cell wall are crucial. The thickness of the cell wall can increase up to three-fold, while the size of cell clumps can increase even more (Zajc et al. 2014). 
In contrast to the large and duplicated genome of $H$. werneckii, the genome of W. ichthyophaga is very compact, with 9.6 $\mathrm{Mb}$ and only 4884 predicted protein-coding genes (Zajc et al 2013). Genome analysis showed that except for the enriched protein family of P-type ATPases, cation transporters are sparse and exhibit low expression levels. Additionally, the expression of most of them is independent of salt concentration. Conversely, a significant expansion of hydrophobins, small amphipathic proteins that reside in the cell wall of filamentous fungi and are involved in a range of processes of cellular growth and development, was found. This significant expansion is specific for W. ichthyophaga, as from the estimated 15 hydrophobin genes found in the genome of a common ancestor of $W$. ichthyophaga, the gene number increased to 26 in halophilic $W$. ichthyophaga (Zajc et al 2013). Compared to those of other fungi, hydrophobins in Wallemia contain a higher proportion of acids on their surface. As this is similar to archaeal halophilic proteins (Madern at al 2000), it is reasonable to suggest that hydrophobins play a role as halophilic proteins. With their acidic amino acids exposed at the surface, they can bind water and salt and facilitate adaptation to salt exposure. Transcriptome analysis supported this idea, as $>50 \%$ of hydrophobins are differentially expressed at different salinities (Zajc et al 2013).

W. ichthyophaga use the strategy of compatible solutes and, as in halotolerant $H$. werneckii, glycerol is the main compatible solute in W. ichthyophaga. The W. ichthyophaga genome contains genes for the enzymes that are involved in glycerol synthesis. The homologue of GPD1, WiGPD1, exhibited lower expression levels and a slower response to hyperosmotic shock in comparison to that of halotolerant $H$. werneckii. As in halotolerant $H$. werneckii, the expression of this gene is regulated by MAP kinase WiHog1 (Konte and Plemenitaš 2013). Genes such as SLT1 for the plasma membrane glycerol/H+ symporter and FPS1, which codes for aquaglyceroporin channel Fps1, were found in multiple copies in W. ichthyophaga, suggesting their involvement in regulating glycerol levels. Among other proteins, the main regulatory enzyme in sterol synthesis, HMG-CoA reductase, also belongs to salt-responsive proteins in W. ichthyophaga and responds with a characteristic salt-dependent U-shape activity pattern (Vaupotič et al 2008). The high activity and protein levels of HMG-CoA reductase in W. ichthyophaga under hypo- and hypersaline conditions suggest that, as in extremely halotolerant $H$. werneckii, HMG-CoA reductase (with $\mathrm{NaCl}$-dependent responses) plays an important role in the physiological adaptation of $W$. ichthyophaga to both hypo- and hyper-saline conditions. 


\section{The HOG signal transduction pathway in the halophilic fungus W. ichthyophaga}

As demonstrated by whole genome analysis (Zajc et al 2013), W. ichthyophaga contains all key genes of the HOG signal transduction pathway (Fig. 2.). Further molecular studies revealed similarities as well as important differences in the molecular machinery involved in sensing and responding to changes in $\mathrm{NaCl}$ concentrations between halophilic $W$. ichthyophaga and halotolerant $H$. werneckii and other fungi. Although many key proteins of the HOG pathway are conserved in halophilic W. ichthyophaga, the architecture and activation of the HOG pathway are specific. While all genes of the HOG components are present in two copies in $H$. werneckii, in $W$. ichthyophaga, all HOG pathway components, except the final MAP kinase Hog 1 (WiHog1 A and B), are present in only one isoform (Konte et al 2013, 2016) .

\subsection{The sensory apparatus of the HOG pathway in W.ichthyophaga}

Compared to S. cerevisiae and halotolerant $H$. werneckii, W. ichthyophaga lacks a considerable number of orthologs that are involved in the osmosensing apparatus of the SHO1 branch of the HOG pathway, e.g., the mucins Msb2 and Hkr1 and the membrane anchor Opy2. Conversely, other orthologs of this branch, e.g., WiCdc24, WiCdc42, WiSte20, WiSte50, and WiSte11, are present (Konte et al. 2016). Despite the presence of proteins of the SHO1 sensory apparatus in halophilic W. ichthyophaga, further studies did not confirm activation of the HOG pathway through this branch. Structural analysis of WiSho1 domains, important for interactions with downstream protein partners, showed that crucial motifs for the interactions with MAPKKK Ste11 MAKK Pbs2 are not conserved in W. ichthyophaga orthologs. Moreover, WiSho1 expressed in $S$. cerevisiae mutant cells did not efficiently complement the function of ScSho1 (Konte et al., 2016)

The core phosphorelay system of the SLN1-like branch is well conserved in ScSho1 in $W$. ichthyophaga (Konte et al 2016). This system contains WiYpd1, WiSsk1, the kinase WiSsk2, and the response regulator WiSkn7. Conversely, no membrane-bound ScSln1 ortholog was found in $W$. ichthyophaga. Instead, as in the genome of halotolerant $H$. werneckii, we confirmed the Group 
III cytosolic histidine kinase WiNik1. The W. ichthyophaga genome contains four hybrid histidine kinases that belong to groups I or II, III, and VIII. It was demonstrated for several fungi that the group III histidine kinases are involved in two-component HOG pathway signaling (Bahn, 2008) . We proposed that the HAMP domain repeats in WiNik1 revealed by structural studies (Konte at all 2016) might be involved in osmosensing in W. ichthyophaga, as has been demonstrated for DhNik1 in moderately halotolerant D. hansenii, CaNik1 in C. albicans and ClNik1 in Candida lusitaniae (andhawa et al., 2016). It has been shown that group III histidine kinases are sensitive to the fungicide fludioxonil, and thus this fungicide represents a potent tool to investigate group III histidine kinase signaling (Furukawa et al. 2012). Our investigation of the sensitivity of $W$. ichthyophaga cells to fludioxonil demonstrated that the presence of fludioxonil completely inhibited the growth of $W$. ichthyophaga cells at all $\mathrm{NaCl}$ concentrations used. This result strongly implies that WiNik1 plays a role in osmosensing machinery.

\subsection{The MAP kinase module of the HOG pathway in W.ichthyophaga}

As MAPKK Pbs2 is the central element of the HOG pathway that can in principle receive hyperosmolarity signals through both the SHO1 and SLN1 branches (Krantz et al., 2006b), we further analyzed WiPbS2, which was identified in the W. ichthyophaga genome. Structural domains, important for interactions with upstream proteins involved in WiPbs2 signal transmission, were compared with $\mathrm{ScPbs} 2$ and the orthologs of some other fungi (Konte et al 2016). It was demonstrated that the proline-rich motif that is crucial for Sho1 binding and signaling through the SHO1 branch in S. cerevisiae (Marles et al., 2004) is not conserved in W. ichthyophaga (Konte et al. 2016). Another important feature of Pbs2 from S. cerevisiae is the docking site for MAPKKK Ste11 (Zarrinpar et al 2004; Tatebayashi et al 2015). Again, in WiPbs2, the Ste11 docking site is only poorly conserved (Konte et al 2016). Poor interactions of WiPbs2 with WiSho1 and WiSte11 indicate that WiPbs2 is not activated via the SHO1 branch, which is in line with the observation that WiSho1 could not complement the function of ScSho1 in the S. cerevisiae mutant strain. Conversely, we found that the ScSsk2/ScSsk22 activation of WiPbs2 is fully conserved (Konte et al 2016). This finding and the sensitivity of W. ichthyophaga cells to fludioxonil highlight the importance of two-component HOG pathway signaling in $W$. ichthyophaga. 
The W. ichthyophaga genome has two gene copies of MAP kinase Hog1, WiHOG A, and WiHOG $B$. Although protein sequence alignment revealed high conservation of the Hog1 kinase domains and motifs in both of the WiHog1A and WiHog1B isoforms, WiHog1A is not fully functional when compared with WiHog1B and ScHog1 in the S. cerevisiae mutant strain (Konte et al 2016). Supported also by the observed lower phosphorylation level of WiHog1 A upon hyperosmotic stress, it was proposed that WiHog1 A cannot optimally interact with the activating MAPKK Pbs2 or its targets. This might be explained by small differences in the ATP-binding site, activation loop, common docking domain, and/or PBD-2 region. Conversely, it was found that WiHog1B was fully phosphorylated; in the S. cerevisiae hogld background, WiHog1B serves as a fully functional kinase. Moreover, WiHog1B also improves the tolerance of the yeast strain to high salinity, which was not observed for HwHog1 expression from the halotolerant $H$. werneckii in $S$. cerevisiae (Lenassi et al., 2007). Additionally, MAPK WiHog upregulated GPD1 transcription, which is important for the synthesis of the main compatible solute, glycerol, in W. ichthyophaga. Again, efficient interaction of the GPD1 promoter was demonstrated with WiHog1B but not WiHog1A (Lenassi et al., 2011, Konte et al 2013).

As in halotolerant $H$. werneckii, WiHog1 transcript levels are salt-dependent (Konte and Plemenitaš, 2013). Interestingly, W. ichthyophaga shows the opposite to the usual phosphorylation pattern found in S. cerevisiae, H. werneckii, and most other studied fungi. In W. ichthyophaga, MAP kinase WiHog1 is constitutively phosphorylated under optimal osmotic conditions (at concentrations of $15-20 \% \mathrm{NaCl}$ ) and is dephosphorylated under both hypo- and hyper-saline conditions. A similar phosphorylation model has so far been reported for pathogenic $C$. neoformans, serotype A, and it was assumed that a unique mechanism of Hog1 phosphorylation in $C$. neoformans is responsible for its higher stress resistance and the virulence of its serotype A (Bahn et al. 2007). Thus, it is reasonable to speculate that, in a similar manner, such HOG pathway regulation might cause the high salt resistance of $W$. ichthyophaga. This phosphorylation pattern also implies that phosphatases play an important role in HOG pathway regulation in $W$. ichthyophaga. The HOG pathway in S. cerevisiae is regulated by negative feedback through the action of phosphothreonine and phosphotyrosine phosphatases (Hohmann et al., 2007). In the W. ichthyophaga genome, we identified the phosphothreonine phosphatase WiPtc1, which is most similar to ScPtc1 and WiPtc3, which is related to ScPtc3 and its paralog ScPtc2 (Warmka et al 
2001, Jacoby et al. 1997). Furthermore, the W. ichthyophaga genome contains two phosphotyrosine phosphatases: WiPtp1 (an ortholog of ScPtp1) and WiPtp3 which is more related to ScPtp3, a broad-range phosphatase in S. cerevisiae (Zajc et al 2013). The presence of these phosphatases in the W. ichthyophaga genome support the importance of WiHog1 dephosphorylation in regulating the HOG signal transduction pathway.

Taken together, our investigation of the architecture of the W. ichthyophaga HOG pathway shows that the interactions of WiPbs2 kinase and the SHO1-branch orthologs are not conserved, which in turn suggests that these orthologs are not involved in WiPbs2 activation. Conversely, the ScSsk2/ScSsk22 activation of WiPbs2 is fully conserved, and W. ichthyophaga cells are sensitive to fludioxonil, which highlights the importance of the two-component HOG pathway signaling in W. ichthyophaga (Konte et al., 2016). A unique WiHog1 phosphorylation pattern was demonstrated to be necessary for the high(er stress-resistance of C. neoformans (Bahn et al., 2007) and could also help W. ichthyophaga to survive in its naturally hypersaline environments.

In this review, the importance of the $\mathrm{HOG}$ pathway in adapting to high $\mathrm{NaCl}$ concentrations in halotolerant $H$. werneckii and the obligate halophile $W$. ichthyophaga is summarized. Although there are many similarities between halotolerant and halophilic fungi, molecular analyses of whole genome sequences of both fungi performed in our laboratory during the past years have revealed important differences in sensing and responding to severe changes in $\mathrm{NaCl}$ concentrations, typical for the natural ecological niche of $H$. werneckii and W. ichthyophaga. We confirmed that the HOG pathway plays an important role in adaptation in both fungi. Studies revealed that the MAP kinase module is conserved in both fungi; however, the mechanisms of activation differ between halotolerant $H$. werneckii and halophilic W. ichthyophaga. In H. werneckii, the HOG pathway is activated via both SHO and SLN branches, whereas in W. ichthyophaga, only the SLN branch appears to be involved. Secondly, HwHog1 is phosphorylated in halotolerant $H$. werneckii and dephosphorylated in halophilic W. ichthyophaga under hypersaline conditions. Molecular investigations of extremely halotolerant $H$. werneckii and halophilic W. ichthyophaga, isolated from the same hypersaline environments, demonstrate that different fungi can employ diverse mechanisms to combat the same harsh conditions. We can also speculate that these differences importantly contribute to the halotolerant and halophilic characteristics ofH.werneckii and W.ichthyophaga . 


\section{References}

Bahn YS, Geunes-Boyer S, Heitman J. Ssk2 mitogen-activated protein kinase kinase kinase governs divergent patterns of the stress-activated Hog1 signaling pathway in Cryptococcus neoformans. Eukaryot. Cell 2007; 6: 2278-2289.

Bahn YS. Master and commander in fungal pathogens: the two-component system and the HOG signaling pathway. Eukaryot Cell 2008; 7:2017-36.

Buh Gašparič M, Lenassi M et al. Insertion of a specific fungal 3'-phosphoadenosine-5'phosphatase motif into a plant homologue improves halotoleranceand drought tolerance of plants. PloS One 2013; 8: e81872.

Butinar L, Sonjak S, Zalar P et al. Melanized halophilic fungi are eukaryotic members of microbial communities in hypersaline waters of solar salterns. Bot Mar 2005; 48:73-9.

Catlett NL, Yoder OC, Turgeon BG.. Whole-genome analysis of two-component signal transduction genes in fungal pathogens. Eukaryot. Cell. 2003; 2:1151-1161.

Fettich M, Lenassi M, Veranič P et al. Identification and characterization of putative osmosensors, HwSho1A and HwSho1B, from the extremely halotolerant black yeast Hortaea werneckii. Fungal Gen Biol 2011; 48:475-84.

Furukawa, K., et al. Aspergillus nidulans HOG pathway is activated only by two-component signalling pathway in response to osmotic stress. Mol Microbiol. 2005; 56:1246-61.

Furukawa, Radhawa A, Kaur H. Fungal fludioxonil sensitivity is diminished by a constitutively active form of the group III histidine kinase.Febs Lett 2012 586:2417-2422

Gorjan A, Plemenitap A. Identification and characterization of ENA ATPases HwENA1 and HwENA2 from the halophilic black yeast Hortaea werneckii. FEMS microbiology letters 2006; 265:41-50.

Gostinčar C, Turk M, Plemenitaš A et al. The expressions of [delta]9-,[delta]12-desaturases and an elongase by the extremely halotolerant Hortaea werneckii are salt dependent. FEMS yeast research 2009; 9: 247-256.

Gostinčar C, Lenassi M, Gunde-Cimerman N et al. Fungal adaptation to extremely high salt concentrations. Adv Appl Microbiol 2011;77:71-96.

Gostinčar C, Stajich J, Kejžar A et al. Seven years at high salinity - experimental evolution of the extremely halotolerant black yeast Hortaea werneckii. Journal of fungi 2021; 7: 1-22. 
Gunde-Cimerman N, Ramos J, Plemenitaš A. Halotolerant and halophilic fungi. Mycol Res 2009;113:1231-41.

Gunde-Cimerman N, Zalar P. Extremely halotolerant and halophilic fungi inhabit brine in solar salterns around the globe. Food Technol Biotechnol 2014; 52:170-9.

Gunde-Cimerman N, Plemenitaš A, Oren A. Strategies of adaptation of microorganisms of the three domains of life to high salt concentrations. FEMS microbiology reviews 2018; 42:353-375.

Han KH, Prade RA.. Osmotic stress-coupled maintenance of polar growth in Aspergillus nidulans. Mol. Microbiol. 2002; 43:1065-1078.

Hohmann S. Osmotic stress signaling and osmoadaptation in yeasts. Microbiol Mol Biol Rev 2002; 66:300-72.

Hohmann S. Control of high osmolarity signalling in the yeast Saccharomyces cerevisiae. FEBS Lett 2009; 583, 4025-29.

Jacob S, Foster A, YemelinA et al. High osmolarity glycerol (HOG) signalling in Magnaporthe oryzae: Identification of MoYPD1 and its role in osmoregulation, fungicide action, and pathogenicity. Fungal Biol. 2015; 119:580-94.

Jacoby T, Flanagan H, Faykin A et al. Two Protein-tyrosine Phosphatases Inactivate the Osmotic Stress Response Pathway in Yeast by Targeting the Mitogen-activated Protein Kinase, Hog1. J Biol Chem 1997; 272:17749-17755.

Jančič S, Nguyen HDT, Frisvad JC et al. A taxonomic revision of the Wallemia sebi species complex. PloS One 2015; 10:e0125933.

Jančic S, Zalar P, Kocev D et al. Halophily reloaded: new insights into the extremophilic lifestyle of Wallemia with the description of Wallemia hederae sp. nov. Fungal Divers 2016; 76:97-118.

Kejžar A, Cibic M, Grötli M, Plemenitaš A et al. The unique characteristics of HOG pathway MAPKs in the extremely halotolerant Hortaea werneckii. FEMS microbiology letters 2015; 362: 1-19.

Kejžar A, Grötli M, Tamás MJ et al. HwHog1 kinase activity is crucial for survival of Hortaea werneckii in extremely hyperosmolar environments. Fungal Genet Biol 2015a; 74:45-58. 
Kogej T, Ramos J, Plemenitaš A et al. The halophilic fungus Hortaea werneckii and the halotolerant fungus Aureobasidium pullulans maintain low intracellular cation concentrations in hypersaline environments. Appl Environ Microbiol 2005; 71:6600-5.

Konte T, Plemenitaš A. The HOG signal transduction pathway in the halophilic fungus Wallemia ichthyophaga: identification and characterisation of MAP kinases WiHog1A and WiHog1B. Extremophiles 2013; 17:623-36.

Konte T, Terpitz U, Plemenitaš A. Reconstruction of the high-osmolarity glycerol (HOG) signaling pathway from the halophilic fungus Wallemia ichtyophaga in Saccharomyces cerevisiae. Frontiers Microbiol 2016; 7:901, DOI: 10.3389/fmicb.2016.00901.

Krantz M, Becit E, Hohmann S. Comparative analysis of HOG pathway proteins to generate hypotheses for functional analysis. Curr. Genet. 2006; 49:152-165

Lenassi M, Plemenitaš A. Novel group VII histidine kinase HwHhk7B from the halophilic fungi Hortaea werneckii has a putative role in osmosensing. Current genetics 2007; 51:393-405.

Lenassi M, Zajc J, Gostinčar C et al. Adaptation of the glycerol-3-phosphate dehydrogenase Gpd1 to high salinities in the extremely halotolerant Hortaea werneckii and halophilic Wallemia ichtyophaga. Fungal Biol 2011;115:959-70.

Lenassi M, Gostinčar C, Jackman S et al. Whole genome duplication and enrichment of metal cation transporters revealed by de novo genome sequencing of extremely halotolerant black yeast Hortaea werneckii. PLoS One 2013; 8:71328.

Madern D, Ebel C, ZaccaiG. Halophilic adaptation of enzymes.Extremophiles 2000; 4:91-98.

Marles JA, Dahesh S, Haynes J et al.l Protein-Protein Interaction Affinity Plays a Crucial Role in Controlling the Sho1p-Mediated Signal Transduction Pathway in Yeast. Molecular Cell 2004;14:813-23

Meena N, Harsimran Kaur H, and Mondal AK Interactions among HAMP Domain Repeats Act as an Osmosensing Molecular Switch in Group III Hybrid Histidine Kinases from Fungi. JBiolChem. 2010; 285:12121-32.

O'Rourke SM, Herskowitz I.. Unique and redundant roles for HOG MAPK pathway components as revealed by whole-genome expression analysis. Mol. Biol. Cell. 2004; 15: 532-542.

Petrovič U, Gunde-Cimerman N, Plemenitaš A. Cellular responses to environmental salinity in the halophilic black yeast Hortaea werneckii. Mol Microbiol 2002; 45:665-72. 
Plemenitaš A, Lenassi M, Konte T et al. Adaptation to high salt concentrations in halotolerant/halophilic fungi: a molecular perspective. Frontiers Microbiol 2014; 5:199, DOI: 10.3389/fmicb.2014.00199.

Plemenitaš A, Konte T, Gostinčar C et al. Transport systems in halophilic fungi. Yeast membrane transport, (Advances in Experimental Medicine and Biology, vol. 892). [S.1.]: Springer. 2016, str. 307-325.

Randhawa A, Chawla S' Mondal AK.Functional dissection of HAMP domains in NIK1 ortholog from pathogenic yeast Candida lusitaniae. Gene 2016; 577:251-57.

Saito H, Posas F. Response to hyperosmotic stress. Genetics 2012; 192:289-318.

Sinha S, Flibotte S, Neira M et al. Insight into the recent genome duplication of the halophilic yeast Hortaea werneckii: combining an improved genome with gene expression and chromatin structure. G3 2017; 7:2015-22.

Srikantha T, Tsai L, Daniels K et al. The two-component hybrid kinase regulator CaNIK1 of Candida albicans Microbiology 1998; 144:2715-29.

Tatebayashi K, Yamamoto K, Nagoya M et al. Osmosensing and scaffolding functions of the oligomeric four-transmembrane domain osmosensor Sho1. Nat Commun 2015; 6:6975

Turk M, Plemenitaš A. The HOG pathway in the halophilic black yeast Hortea werneckii: isolation of the HOG1 homolog gene and activation of HwHog1p. FEMS microbiology letters 2002; 216: 193-99.

Turk M, Méjanelle L, Šentjurc M et al. Salt-induced changes in lipid composition and membrane fluidity of halophilic yeast-like melanized fungi. Extremophiles 2004; 8:53-61.

Turk M, Plemenitaš A, Gunde-Cimerman N. Extremophilic yeasts: plasma-membrane fluidity as determinant of stress tolerance. Fungal Biol 2011; 115:950-958.

Vaupotič T, Plemenitaš A. Osmoadaptation-dependent activity of microsomal HMG-CoA reductase in the extremely halotolerant black yeast Hortaea werneckii is regulated by ubiquitination. FEBS letters 2007; 581:3391-3395.

Vaupotič T, Gunde-Cimrman N, Plemenitaš A.Ana. Novel 3'-phosphoadenosine-5'phosphatases from extremely halotolerant Hortaea werneckii reveal insight into molecular determinants of salt tolerance of black yeasts. Fungal genetics and biology 2007a; 44: 1109-1122. 
Vaupotič T, Plemenitaš A. Differential gene expression and HogI interaction with osmoresponsive genes in the extremely halotolerant black yeast Hortaea werneckii. BMC Genomics 2007b; 8:280, DOI: 10.1186/1471-2164-8-280.

Vaupotič T, Veranič P, Petrovič U et al. HMG-CoA reductase is regulated by environmental salinity and its activity is essential for halotolerance in halophilic fungi. Studies in mycology 2008; 61:61-66.

Vaupotic T, Veranic P, Jenoe P et al. Mitochondrial mediation of environmental osmolytes discrimination during osmoadaptation in the extremely halotolerant black yeast Hortaea werneckii. Fungal Genet Biol 2008a; 45:994-1007.

Warmka J, J Hanneman J, Lee J et al. Ptc1, a type 2C Ser/Thr phosphatase, inactivates the HOG pathway by dephosphorylating the mitogen-activated protein kinase Hog1 Mol Cell Biol 2001; 21:51-60.

Zajc J, Liu Y, Dai W et al. Genome and transcriptome sequencing of the halophilic fungus Wallemia ichthyophaga: haloadaptations present and absent. BMC Genomics 2013; 14:617, DOI: 10.1186/1471-2164-14-617.

Zajc J, Kogej T, Galinski EA et al. Osmoadaptation strategy of the most halophilic fungus, Wallemia ichthyophaga, growing optimally at salinities above $15 \% \mathrm{NaCl}$. Appl Environ Microbiol 2014; 80:247-56.

Zarrinpar A, Bhattacharyy RP, Nittler MP. Sho1 and Pbs2 Act as Coscaffolds Linking Components in the Yeast High Osmolarity MAP Kinase Pathway. Mol.Cell 2004; 6: 825832.

Figure 1: Model of the HOG pathway architecture in H.werneckii. Putative HOG-pathway components identified in the genome of H.werneckii are shown. HwShol and kinases Hw HhK, HwSte 11, HwPbs2 and HwHog 1 proteins were further characterized as summarized in the text. Shown are also identified Hog1 target osmoresponsive genes. (Illustration made by Matej Kocjan)

Figure 2: Model of the HOG pathway architecture in W. ichthyophaga. Putative HOGpathway components identified in the genome of W. ichthyophaga are shown. WiSho1, WiSte11, 
WiPbs2 and WiHog1proteins were characterized in details as summarized in the text. Identified target genes of WiHog1, WiGpp1 and Wipd1are shown. (Illustration made by Matej Kocjan)

\section{Funding}

This study was funded by Research Grant P1-0170, and in part by a Young Researcher Fellowships from the Slovenian Research Agency.

\section{Conflict of Interest Statement}

The author declare that the research was conducted in the absence of any commercial or financial relationships that could be construed as a potential conflict of interest. 